November 1999

\title{
Finite gravitational action for higher derivative and stringy gravities
}

\author{
ShIn'ICHI NOJIRI and SERGEI D. ODINTSOV \\ Department of Mathematics and Physics \\ National Defence Academy, Hashirimizu Yokosuka 239, JAPAN \\ A Tomsk Pedagogical University, 634041 Tomsk, RUSSIA
}

\begin{abstract}
We generalize the local surface counterterm prescription suggested in Einstein gravity for higher derivative (HD) and Weyl gravities. Explicitly, the surface counterterm is found for three- and five-dimensional HD gravities. As a result, the gravitational action for asymptotically AdS spaces is finite and gravitational energy-momentum tensor is well-defined. The holographic trace anomaly for d2 and d4 boundary (gauge) QFT dual to above HD gravity is calculated from gravitational energy-momentum tensor. The calculation of AdS black hole mass in HD gravity is presented within above prescrition. The comparison with the standard prescription (using reference spacetime) is done.
\end{abstract}

\footnotetext{
${ }^{1}$ e-mail: nojiri@cc.nda.ac.jp, snojiri@yukawa.kyoto-u.ac.jp

${ }^{2}$ e-mail: odintsov@mail.tomsknet.ru, odintsov@itp.uni-leipzig.de
} 


\section{Introduction}

One of the important achievements in AdS/CFT correspondence (for a general review and list of references, see [1]) is related with the possibility to make the the finite gravitational action and to find the quasilocal stress tensor in gravity using a local surface counterterm prescription [2]. With such prescription one chooses the coordinate invariant functional of the intrinsic boundary geometry and adds it to gravitational action. As a result, the equations of motion are not modified. However, the specific choice of this functional (often called the surface counterterm) cancels the divergences of gravitational action on the background under consideration. Then, one comes to well-defined expression for gravitational energy-momentum tensor and action [2].

From the very beginning, the local counterterm prescription looks more elegant than the standard prescription of subtraction of reference spacetime. There, the action is regulated by restriction of spacetime to the interior of some boundary geometry and then, by subtraction of (infinite) gravitational action of another (reference) background which has the same boundary [3]. Clearly, the standard prescription is ambiguous as it depends from the choice of reference spacetime. Moreover, it is not always possible to select the necessary reference background. Very often the embedding of boundary geometry in the reference spacetime may be done only approximately. Nevertheless, in many cases the standard prescription works and finite gravitational action is obtained in the limit of infinite boundary. The quasilocal stress tensor could be then found by variation of finite gravitational action over surface metric [曰].

The local surface counterterms prescription [2] has been successfully tested in asymptotically AdS spacetimes. It reproduces correctly the mass and angular momentum for various AdS backgrounds. The results coincide with the ones for same background (say, energy evaluation [5, 6, 7, 8, 9, 10]) using standard procedure. Explicitly, the surface counterterms (finite polynomials) for $d \leq 4, d \leq 6, d \leq 8$ asymptotically AdS spacetimes have been found in refs. 22, 11, 12 respectively.

It is very interesting that such prescription has clear similarity (via AdS/CFT correspondence) with usual counterterms prescription (renormalization) in quantum field theory. Moreover, as it was shown in ref. 113 (see also ref.[14]) the local surface counterterm prescription admits the natural 
continuation for asymptotically flat spaces.

So far, the consideration of above prescription has been limited to Einstein gravity where the universal and widely accepted Gibbons-Hawking boundary term [3] is known. The role of this term is to make the variational procedure to be well-defined one.

It would be extremely interesting to test the universality of prescription [2] applying it to another gravitational theories. The purpose of this work is to formulate the local counterterm prescription for higher derivative (or $R^{2}$ ) gravity. As is known such theory is good alternative for Einstein gravity. Moreover, it could be that higher derivative (HD) terms are relevant only near Planck scale as there may be mechanisms to make such HD terms negligible at present stage of Universe evolution. Such theory is well studied as perturbative QFT (for a review, see [15]). HD gravity has better ultraviolet properties than Einstein one. From another side, HD gravity often appears as low-energy effective action from string theory. Hence, it may be considered as stringy gravity. The important difference of HD gravity from Einstein gravity is the fact that boundary term (analog of Gibbons-Hawking term) is not only much more complicated but also there is no standard form of such term accepted in the literature. The reason is that boundary term also includes the HD terms. Hence, it is quite difficult to make the variational procedure for HD gravity to be well-defined (unlike the case of Einstein gravity).

In the next section we discuss the boundary term in HD gravity and in its special version (Weyl or stringy) gravity. The local surface counterterm in such theories is explicitly found for $\mathrm{d} 2$ and $\mathrm{d} 4$ cases, i.e. when the corresponding gravity theory (bulk) is formulated in three and five dimensions. Comparison with the Einstein gravity is made. As a result the finite gravitational action for HD gravity is found as well as gravitational energymomentum tensor. From this as an explicit check we define the holographic conformal anomaly in d2 and d4 (via AdS/CFT correspondence). This conformal anomaly found from finite gravitational action in three and five dimensions coincides with the previous calculation of holographic conformal anomaly using other methods. In third section the calculation of AdS black hole mass is done for HD gravity. In this calculation the local surface counterterm prescription is used. It is shown that the result coincides with the corresponding calculation with the help of standard prescription. Sometimes, there may appear some finite difference between the calculation in two differ- 
ent prescriptions. This difference is regarded as regularization dependence. We show how one can make two different results coinciding by fixing of some arbitrary parameters in local surface counterterm for HD gravity. In last section the short summary is given.

\section{Surface counterterm and finite gravita- tional action}

In the present section we will define the local surface counterterm for HD gravity in three and five dimensions. This will make the corresponding gravitational action for asymptotically AdS spaces to be finite. We start with the action:

$$
S=\int_{M_{d+1}} d^{d+1} x \sqrt{-\hat{G}}\left\{a \hat{R}^{2}+b \hat{R}_{\mu \nu} \hat{R}^{\mu \nu}+c \hat{R}_{\mu \nu \xi \sigma} \hat{R}^{\mu \nu \xi \sigma}+\frac{1}{\kappa^{2}} \hat{R}-\Lambda\right\} .
$$

Here $M_{d+1}$ is $d+1$ dimensional manifold whose boundary is $d$ dimensional manifold $M_{d}$. The conventions of curvatures are given by

$$
\begin{aligned}
R & =g^{\mu \nu} R_{\mu \nu} \\
R_{\mu \nu} & =-\Gamma_{\mu \lambda, \kappa}^{\lambda}+\Gamma_{\mu \kappa, \lambda}^{\lambda}-\Gamma_{\mu \lambda}^{\eta} \Gamma_{\kappa \eta}^{\lambda}+\Gamma_{\mu \kappa}^{\eta} \Gamma_{\lambda \eta}^{\lambda} \\
\Gamma_{\mu \lambda}^{\eta} & =\frac{1}{2} g^{\eta \nu}\left(g_{\mu \nu, \lambda}+g_{\lambda \nu, \mu}-g_{\mu \lambda, \nu}\right) .
\end{aligned}
$$

When $a=b=c=0$, the action (11) becomes that of the Einstein gravity:

$$
S=\int_{M_{d+1}} d^{d+1} x \sqrt{-\hat{G}}\left\{\frac{1}{\kappa^{2}} \hat{R}-\Lambda\right\}
$$

If we choose

$$
a=\frac{2}{d(d-1)} \hat{c}, \quad b=-\frac{4}{d-1} \hat{c}, \quad c=\hat{c}
$$

the HD part of action is given by the square of the Weyl tensor $C_{\mu \nu \rho \sigma}$ :

$$
S=\int_{M_{d+1}} d^{d+1} x \sqrt{-\hat{G}}\left\{\hat{c} \hat{C}_{\mu \nu \xi \sigma} \hat{C}^{\mu \nu \xi \sigma}+\frac{1}{\kappa^{2}} \hat{R}-\Lambda\right\}
$$

The string theory dual to $\mathcal{N}=2$ superconformal field theory is presumably IIB string on $\mathrm{AdS}_{5} \times X_{5}$ [16] where $X_{5}=S^{5} / Z_{2}$. (The $\mathcal{N}=2 S p(N)$ theory 
arises as the low-energy theory on the world volume on $N$ D3-branes sitting inside 8 D7-branes at an O7-brane). Then in the absence of Weyl term, $\frac{1}{\kappa^{2}}$ and $\Lambda$ are given by

$$
\frac{1}{\kappa^{2}}=\frac{N^{2}}{4 \pi^{2}}, \quad \Lambda=-\frac{12 N^{2}}{4 \pi^{2}} .
$$

This defines the bulk gravitational theory dual to suer YM theory with two supersymmetries. The Riemann curvature squared term in the above bulk action may be deduced from heterotic string via heterotic-type I duality [17]. Using field redefinition ambiguity [18] one can suppose that there exists the scheme where $R_{\mu \nu \alpha \beta}^{2}$ may be modified to $C_{\mu \nu \alpha \beta}^{2}$ in the same way as in ref. [19]. Then, the action (3) is presumably the bulk action dual to $\mathcal{N}=2$ SCFT. In [20], for the $\mathcal{N}=2$ theory with the gauge group $S p(N)$, the usual UV Weyl anomaly via AdS/CFT correspondence is reproduced if

$$
\tilde{c}=\frac{6 N-1}{24 \cdot 16 \pi^{2}}, \quad \frac{1}{\kappa^{2}}=\frac{12 N^{2}+12 N-1}{3 \cdot 16 \pi^{2}} .
$$

The second equation in (7) is, however, not compatible with (6), where $\frac{1}{\kappa^{2}}=$ $\frac{N^{2}}{4 \pi^{2}}$. This might suggest that some sub-leading corrections to $\frac{1}{\kappa^{2}}$ and/or $\Lambda$ would be necessary. The explicit form of such sub-leading terms has been defined in ref. 20. We call the model corresponding to (5) as stringy gravity.

When $a, b$ and $c$ are given by

$$
a=c=\hat{a}, \quad b=-4 \hat{a}
$$

for $d=3$, the $R^{2}$ terms in (11) form the Gauss-Bonnet invariant $\tilde{G}$ :

$$
S=\int_{M_{d+1}} d^{d+1} x \sqrt{-\hat{G}}\left\{\hat{a} \tilde{G}+\frac{1}{\kappa^{2}} \hat{R}-\Lambda\right\} .
$$

The variation of the action (1) with respect to $\hat{G}^{\mu \nu}$ is given by

$$
\begin{aligned}
\delta S= & \delta S_{M_{d+1}}+\delta S_{M_{d}} \\
\delta S_{M_{d+1}}= & \int_{M_{d+1}} d^{d+1} x \sqrt{-\hat{G}} \delta \hat{G}^{\zeta \xi}\left[-\frac{1}{2} G_{\zeta \xi}\left\{a \hat{R}^{2}+b \hat{R}_{\mu \nu} \hat{R}^{\mu \nu}\right.\right. \\
& \left.+c \hat{R}_{\mu \nu \xi \sigma} \hat{R}^{\mu \nu \xi \sigma}+\frac{1}{\kappa^{2}} \hat{R}-\Lambda\right\} \\
& \left.+2 a R R_{\zeta \xi}+2 b \hat{R}_{\mu \zeta} \hat{R}_{\xi}^{\mu}+2 c \hat{R}_{\zeta \mu \nu \xi} \hat{R}_{\xi}^{\mu \nu \xi}+\frac{1}{\kappa^{2}} \hat{R}_{\zeta \xi}+\cdots\right]
\end{aligned}
$$




$$
\begin{aligned}
\delta S_{M_{d}}= & \int_{M_{d}} d^{d} x \sqrt{-\hat{g}} n_{\mu}\left[2 a \hat{R}\left\{\partial^{\mu}\left(\hat{G}_{\xi \nu} \delta \hat{G}^{\xi \nu}\right)-D_{\nu}\left(\delta \hat{G}^{\mu \nu}\right)\right\}\right. \\
& +2 b\left\{\frac{1}{2} \hat{R}^{\mu \nu} \partial_{\nu}\left(\hat{G}_{\xi \sigma} \delta \hat{G}^{\xi \sigma}\right)-\hat{R}_{\nu \sigma} D^{\sigma}\left(\delta \hat{G}^{\mu \nu}\right)\right. \\
& \left.+\frac{1}{2} \hat{R}_{\xi \sigma} D^{\mu}\left(\delta \hat{G}^{\xi \sigma}\right)\right\}+4 c \hat{R}^{\mu \nu \xi \sigma} D_{\xi}\left(\hat{G}_{\nu \tau} \hat{G}_{\sigma \kappa} \delta \hat{G}^{\tau \kappa}\right) \\
& \left.+\frac{1}{\kappa^{2}}\left\{\partial^{\mu}\left(\hat{G}_{\xi \nu} \delta \hat{G}^{\xi \nu}\right)-D_{\nu}\left(\delta \hat{G}^{\mu \nu}\right)\right\}\right] .
\end{aligned}
$$

Here $\cdots$ in $\delta S_{M_{d+1}}$ expresses the terms containing the covariant derivative of the curvatures, $\hat{g}_{\mu \nu}$ is the metric on $M_{d}$ induced from $\hat{G}_{\mu \nu}$ and $n_{\mu}$ is the unit vector normal to $M_{d}$. We now choose the metric in the following form:

$$
d s^{2} \equiv \hat{G}_{\mu \nu} d x^{\mu} d x^{\nu}=\frac{l^{2}}{4} \rho^{-2} d \rho d \rho+\sum_{i=1}^{d} \hat{g}_{i j} d x^{i} d x^{j}, \quad \hat{g}_{i j}=\rho^{-1} g_{i j}
$$

Then $n^{\mu}$ and its covariant derivatives are given by,

$$
n^{\mu}=\left(\frac{2 \rho}{l}, 0, \cdots, 0\right), \quad D_{\rho} n^{\rho}=D_{\rho} n^{i}=D_{i} n^{\rho}=0, \quad D_{i} n^{j}=\frac{\rho}{l} \hat{g}^{i k} \hat{g}_{k j}^{\prime} .
$$

Here $^{\prime} \equiv \frac{\partial}{\partial_{\rho}}$. In the coordinate choice (11), the surface terms $\delta S_{M_{d}}$ in (10) have the following form

$$
\begin{aligned}
\delta S_{M_{d}}= & \lim _{\rho \rightarrow 0} \int_{M_{d}} d^{d} x \sqrt{-\hat{g}} \frac{2 \rho}{l}\left[2 a \hat{R}\left(\hat{g}_{i j} \delta \hat{g}^{i j}\right)^{\prime}\right. \\
& +b\left\{\hat{R}_{\rho}{ }^{\rho} \partial_{\rho}\left(\hat{g}_{i j} \delta \hat{g}^{i j}\right)^{\prime}+\hat{R}_{i j}\left(\delta \hat{g}^{i j}{ }^{\prime}+\hat{g}^{i k} \hat{g}_{k l} \delta \hat{g}^{l j}\right)\right\} \\
& \left.+4 c \hat{R}^{\rho}{ }_{i j}{ }_{j}\left(\delta \hat{g}^{i j}{ }^{\prime}+\hat{g}^{i k} \hat{g}_{k l} \delta \hat{g}^{l j}\right)+\frac{1}{\kappa^{2}} \partial^{\rho}\left(\hat{g}_{i j} \delta \hat{g}^{i j}\right)\right] .
\end{aligned}
$$

Note that the terms containing $\delta \hat{g}^{\rho \rho}$ or $\delta \hat{g}^{\rho i}$ vanish. The variation $\delta S_{M_{d}}$ contains the derivative of $\delta \hat{g}^{i j}$ with respect to $\rho$, which makes the variational principle ill-defined. In order that the variational principle is well-defined on the boundary, the variation of the action should be written in the form of

$$
\delta S_{M_{d}}=\lim _{\rho \rightarrow 0} \int_{M_{d}} d^{d} x \sqrt{-\hat{g}} \delta \hat{g}^{i j}\{\cdots\}
$$

after using the partial integration. If we put $\{\cdots\}=0$ for $\{\cdots\}$ in (14), we could obtain the boundary condition. If the variation of the action on 
the boundary contains $\left(\delta \hat{g}^{i j}\right)^{\prime}$, however, we cannot partially integrate it with respect to $\rho$ on the boundary to rewrite the variation in the form of (14) since $\rho$ is the coordinate expressing the direction perpendicular to the boundary. Therefore the "minimum" of the action is ambiguous. Such a problem was well studied in [3] for the Einstein gravity $(a=b=c=0)$ and the boundary term was added to the action, which cancels the variation :

$$
S_{b}^{\mathrm{GH}}=-\frac{2}{\tilde{\kappa}^{2}} \int_{M_{d}} d^{d} x \sqrt{-\hat{g}} D_{\mu} n^{\mu} .
$$

In the coordinate choice (11), the action (15) has the form

$$
S_{b}^{\mathrm{GH}}=-\frac{2}{\tilde{\kappa}^{2}} \int_{M_{d}} d^{d} x \sqrt{-\hat{g}} \frac{\rho}{l} \hat{g}_{i j}\left(\hat{g}_{i j}\right)^{\prime}
$$

Then the variation over the metric $\hat{g}_{i j}$ gives

$\delta S_{b}^{\mathrm{GH}}=-\frac{2}{\tilde{\kappa}} \int_{M_{d}} d^{d} x \sqrt{-\hat{g}} \frac{\rho}{l}\left[\delta \hat{g}^{i j}\left\{-\hat{g}_{i k} \hat{g}_{i l}\left(\hat{g}_{k l}\right)^{\prime}-\frac{1}{2} \hat{g}_{i j} \hat{g}_{k l}\left(\hat{g}_{k l}\right)^{\prime}\right\}+\hat{g}_{i j}\left(\delta \hat{g}_{i j}\right)^{\prime}\right]$.

From the other side, the surface terms in the variation of the bulk Einstein action $(a=b=c=0$ in (13)) have the form

$$
\delta S_{M_{d}}^{\text {Einstein }}=\lim _{\rho \rightarrow 0} \frac{1}{\kappa^{2}} \int_{M_{d}} d^{d} x \sqrt{-\hat{g}} \frac{2 \rho}{l}\left[\hat{g}_{i j}^{\prime} \delta \hat{g}^{i j}+\hat{g}_{i j}\left(\delta \hat{g}^{i j}\right)^{\prime}\right] .
$$

Then we find the terms containing $\left(\delta \hat{g}^{i j}\right)^{\prime}$ in $(17)$ and (18) are cancelled with each other.

For HD gravity with the square of the scalar curvature and the square of the Weyl tensor $C_{\mu \nu \rho \sigma}$, where the action is given by

$$
S=\int_{M_{d+1}} d^{d+1} x \sqrt{-\hat{G}}\left\{a \hat{R}^{2}+\hat{c} \hat{C}_{\mu \nu \xi \sigma} \hat{C}^{\mu \nu \xi \sigma}\right\}
$$

the boundary terms were proposed in 21] as follows:

$$
\begin{aligned}
S_{b}^{\mathrm{HL}}= & \int_{M_{d}} d^{d} x \sqrt{-\hat{g}}\left[4\left(a+\frac{2 \hat{c}}{d(d-1)}\right) \hat{R} D_{\mu} n^{\mu}\right. \\
& -\frac{8 \hat{c}}{d-1}\left(n_{\mu} n_{\nu} \hat{R}^{\mu \nu} D_{\sigma} n^{\sigma}+\hat{R}_{\mu \nu} D^{\mu} n^{\nu}\right) \\
& \left.+8 \hat{c} n_{\mu} n_{\nu} \hat{R}^{\mu \tau \nu \sigma} D_{\tau} n_{\sigma}\right] .
\end{aligned}
$$


For general $R^{2}$ gravity (1), if the curvatures would not contain $\left(\hat{g}_{i j}\right)^{\prime}$ nor $\left(\hat{g}_{i j}\right)^{\prime \prime}$, which appear in $\delta S_{M_{d}}$ of (10), the boundary term would be given by

$$
\begin{aligned}
S_{b}^{R^{2}}= & \int_{M_{d}} d^{d} x \sqrt{-\hat{g}}\left[4 a \hat{R} D_{\mu} n^{\mu}+2 b\left(n_{\mu} n_{\nu} \hat{R}^{\mu \nu} D_{\sigma} n^{\sigma}+\hat{R}_{\mu \nu} D^{\mu} n^{\nu}\right)\right. \\
& \left.+8 c n_{\mu} n_{\nu} \hat{R}^{\mu \tau \nu \sigma} D_{\tau} n_{\sigma}-\frac{2}{\tilde{\kappa}^{2}} D_{\mu} n^{\mu}\right] .
\end{aligned}
$$

If we choose $a \rightarrow a+\frac{2}{d(d-1)} \hat{c}, b \rightarrow-\frac{4}{d-1} \hat{c}, c \rightarrow \hat{c}$ (see Eq.(4)), the boundary terms for the action with the squares of the scalar curvature and the Weyl tensor correspond to the boundary action (20) in [21]. In the coordinate choice (11), $S_{b}^{R^{2}}$ in (21) has the following form:

$$
\begin{aligned}
S_{b}^{R^{2}}= & \lim _{\rho \rightarrow 0} \int_{M_{d}} d^{d} x \sqrt{-\hat{g}} \frac{\rho}{l}\left[-4 a \hat{R} \hat{g}_{i j}\left(\hat{g}^{i j}\right)^{\prime}-2 b\left(\hat{R}_{\rho}^{\rho} \hat{g}_{i j}\left(\hat{g}^{i j}\right)^{\prime}+\hat{R}_{i j}\left(\hat{g}^{i j}\right)^{\prime}\right)\right. \\
& \left.+8 c \hat{R}_{i \rho j}^{\rho}\left(\hat{g}^{i j}\right)^{\prime}-\frac{2}{\tilde{\kappa}^{2}} \hat{g}_{i j}\left(\hat{g}^{i j}\right)^{\prime}\right] .
\end{aligned}
$$

In the coordinate choice (11), the curvatures appearing in (22) have the following forms:

$$
\begin{aligned}
R & =\rho R+\frac{3 \rho^{2}}{l^{2}} \hat{g}^{i j} \hat{g}^{k l} \hat{g}_{i k}^{\prime} \hat{g}_{j l}^{\prime}-\frac{4 \rho^{2}}{l^{2}} \hat{g}^{i j} \hat{g}_{i j}^{\prime \prime}-\frac{\rho^{2}}{l^{2}} \hat{g}^{i j} \hat{g}^{k l} \hat{g}_{i j}^{\prime} \hat{g}_{k l}^{\prime} \\
R_{\rho \rho} & =\frac{1}{4} \hat{g}^{i j} \hat{g}^{k l} \hat{g}_{i k}^{\prime} \hat{g}_{j l}^{\prime} \\
R_{i j} & =R_{i j}+\frac{2 \rho^{2}}{l^{2}} \hat{g}^{k l} \hat{g}_{i k}^{\prime} \hat{g}_{j l}^{\prime}-\frac{2 \rho^{2}}{l^{2}} \hat{g}_{i j}^{\prime \prime}-\frac{\rho^{2}}{l^{2}} \hat{g}^{k l} \hat{g}_{i j}^{\prime} \hat{g}_{k l}^{\prime} \\
R_{\rho i \rho j} & =\frac{1}{4} \hat{g}^{k l} \hat{g}_{i k}^{\prime} \hat{g}_{j l}^{\prime}-\frac{1}{2} \hat{g}_{i j}^{\prime \prime} .
\end{aligned}
$$

Here $R$ and $R_{i j}$ are scalar and the Ricci curvatures given by $g_{i j}$ in (11). We should note that the curvatures contain $\left(\hat{g}_{i j}\right)^{\prime}$ and/or $\left(\hat{g}_{i j}\right)^{\prime \prime}$. Therefore the variation of the curvatures in the surface terms in (21) or (22) induces new terms containing $\left(\delta \hat{g}^{i j}\right)^{\prime}$ and $\left(\delta \hat{g}^{i j}\right)^{\prime \prime}$. Therefore we need to add more surface terms to cancel them. It seems very difficult to cancel the terms containing $\left(\delta \hat{g}^{i j}\right)^{\prime \prime}$. Instead of (21), we choose $S_{b}$ by new parameters $\tilde{a}, \tilde{b}, \tilde{c}$ and $\tilde{\kappa}$ in the following form:

$$
S_{b}=S_{b}^{(1)}+S_{b}^{(2)}
$$




$$
\begin{aligned}
S_{b}^{(1)=} & \int_{M_{d}} d^{d} x \sqrt{-\hat{g}}\left[4 \tilde{a} \hat{R} D_{\mu} n^{\mu}+2 \tilde{b}\left(n_{\mu} n_{\nu} \hat{R}^{\mu \nu} D_{\sigma} n^{\sigma}+\hat{R}_{\mu \nu} D^{\mu} n^{\nu}\right)\right. \\
& \left.+8 \tilde{c} n_{\mu} n_{\nu} \hat{R}^{\mu \tau \nu \sigma} D_{\tau} n_{\sigma}-\frac{2}{\tilde{\kappa}^{2}} D_{\mu} n^{\mu}\right] \\
S_{b}^{(2)=} & \int_{M_{d}} d^{d} x \sqrt{-\hat{g}}[\xi \tilde{R}+\eta] .
\end{aligned}
$$

Here $\tilde{R}$ is the scalar curvature on the boundary and is defined via $\hat{g}_{i j}$ in (11). In (24), $S_{b}^{(1)}$ is a generalization of (15) in Einstein gravity and corresponds to (21). We also need to add $S_{b}^{(2)}$ (surface counterterm) which cancels the divergence appearing in the limit of $\rho \rightarrow 0$ in the action. The divergence makes the energy-momentum tensor ill-defined. Note that $S_{b}^{(2)}$ is only given in terms of the boundary quantities, which do not affect the variational principle. If $\tilde{\kappa}=\kappa$, the term corresponding to $-\frac{2}{\tilde{\kappa}} D_{\mu} n^{\mu}$ is the term found in [3]. If we choose $\tilde{a}=a+\frac{2}{d(d-1)} \hat{c}, \tilde{b}=-\frac{4}{d-1} \hat{c}, \tilde{c}=\hat{c}$ as in Weyl gravity (see Eq.(四)), the boundary terms correspond to (20). Since it is difficult to determine the coefficients $\tilde{a}, \tilde{b}, \tilde{c}$ and $\tilde{\kappa}$ by the variational principle in a closed form, we determine the coefficients $\tilde{a}, \tilde{b}, \tilde{c}$ and $\tilde{\kappa}$ and also $\eta$ and $\xi$ simultaneously from the condition of the cancellation of the divergences. If $d \leq 4$, the terms in (24) are enough to well-define the variational principle in the asymptotically AdS background. As we define these coefficients from the condition of cancellation of the divergence, not only $S_{b}^{(2)}$ will be surface counterterm but also part of surface counterterm will be included in $S_{b}^{(1)}$. It is difficult to divide $S_{b}^{(1)}$ to boundary term and surface counterterm (and such division even looks unnatural as same terms with different coefficients appear from both parts of surface terms) so we keep this term mixed. The important thing will be that it leads to finite gravitational action for asymptotically AdS spaces.

In the coordinate choice (11), $S_{b}$ in (24) has the following form:

$$
\begin{aligned}
S_{b}= & \lim _{\rho \rightarrow 0} \int_{M_{d}} d^{d} x \sqrt{-\hat{g}} \frac{\rho}{l}\left[-4 \tilde{a} \hat{R} \hat{g}_{i j}\left(\hat{g}^{i j}\right)^{\prime}-2 \tilde{b}\left(\hat{R}_{\rho}^{\rho} \hat{g}_{i j}\left(\hat{g}^{i j}\right)^{\prime}+\hat{R}_{i j}\left(\hat{g}^{i j}\right)^{\prime}\right)\right. \\
& \left.+8 \tilde{c} \hat{R}_{i \rho j}^{\rho}\left(\hat{g}^{i j}\right)^{\prime}-\frac{2}{\tilde{\kappa}^{2}} \hat{g}_{i j}\left(\hat{g}^{i j}\right)^{\prime}+\frac{l}{\rho}(\xi \tilde{R}+\eta)\right] .
\end{aligned}
$$

We now determine the coefficients $\tilde{a}, \tilde{b}, \tilde{c}$ and $\tilde{\kappa}$ and also $\eta$ and $\xi$ to cancel the divergent parts in the bulk action (11). The divergent parts, except the 
logarithmically divergent parts, in the bulk action is given by

$$
\begin{aligned}
S= & \int d^{d+1} x \frac{l \rho^{-\frac{d}{2}-1}}{2} \sqrt{-g_{(0)}}\left[\frac{4 d^{3}+4 d^{2}}{l^{4}} a+\frac{4 d^{2}}{l^{4}} b+\frac{8 d}{l^{4}} c-\frac{2 d}{l^{2} \kappa^{2}}\right. \\
& +\rho\left\{R_{(0)}\left(-\frac{2 d(d+1)}{l^{2}} a-\frac{2 d}{l^{2}} b-\frac{4}{l^{2}} c+\frac{1}{\kappa^{2}}\right)\right. \\
& +g_{(0)}^{i j} g_{(1) i j}\left(\frac{-4 d^{3}+4 d^{2}+8 d}{2 l^{4}} a+\frac{-4 d^{2}+8 d}{2 l^{4}} b+\frac{-4 d+8}{l^{4}} c\right. \\
& \left.\left.\left.+\frac{2 d-4}{2 l^{2} \kappa^{2}}\right)\right\}+\mathcal{O}\left(\rho^{2}\right)\right] .
\end{aligned}
$$

Here we expand the metric $g_{i j}$ as a power series with respect to $\rho$,

$$
g_{i j}=g_{(0) i j}+\rho g_{(1) i j}+\rho^{2} g_{(2) i j}+\cdots
$$

and the radius $l$ of AdS is found by solving the equation

$$
0=\frac{d^{4}-2 d^{3}-3 d^{2}}{l^{4}} a+\frac{d^{3}-3 d^{2}}{l^{4}} b+\frac{2 d^{2}-6 d}{l^{4}} c-\frac{d^{2}-d}{\kappa^{2} l^{2}}-\Lambda,
$$

which follows from eqs. of motion. In Eq.(26), $\Lambda$ is deleted by using (28).

Especially the term proportional to $g_{(0)}^{i j} g_{(1) i j}$ in (26) would break the principle of the holography, where only $g_{(0) i j}$, which is the boundary value of the metric tensor $g_{i j}$, should give the boundary condition but $g_{(1) i j}$, which is the first order derivative of $g_{i j}$ with respect to $\rho$, should not do. If we treat $g_{(1) i j}$ as dynamical variable, we obtain the equation $g_{(0) i j}=0$ from the variation of $g_{(1) i j}$, which is inconsistent.

For $d=4$ there also appear the following logarithmically divergent terms:

$$
\begin{aligned}
S_{\ln }= & \int d^{d+1} x \frac{l \rho^{-\frac{d}{2}+1}}{2} \sqrt{-g_{(0)}}\left[a R_{(0)}^{2}+b R_{(0) i j} R_{(0)}^{i j}+c R_{(0) i j k l} R_{(0)}^{i j k l}\right. \\
& +R_{(0)}^{i j} g_{(1) i j}\left(l^{2} K+\frac{2 d-4}{l^{2}} b\right)-3(d-4) K g_{(0)}^{i j} g_{(2) i j} \\
& +R_{(0)} g_{(0)}^{i j} g_{(1) i j}\left(-\frac{l^{2}}{2} K+\frac{4 d-4}{l^{2}} a+\frac{2}{l^{2}} b+\frac{4}{l^{2}} c\right) \\
& +g_{(0)}^{i j} g_{(0)}^{k l} g_{(1) i k} g_{(1) j l}\left(\frac{3 d-10}{2} K+\frac{4 d^{2}-16 d+16}{4 l^{4}} b+\frac{4 d-8}{l^{4}} c\right) \\
& \left.+\left(g_{(0)}^{i j} g_{(1) i j}\right)^{2}\left(-\frac{3 d-8}{4} K+\frac{4 d^{2}-8 d+4}{l^{4}} a+\frac{3 d-4}{l^{4}} b+\frac{4}{l^{4}} c\right)\right] .
\end{aligned}
$$


Here

$$
K \equiv \frac{2 d^{2}+2 d}{l^{4}} a+\frac{2 d}{l^{4}} b+\frac{4}{l^{4}} c-\frac{1}{l^{2} \kappa^{2}} .
$$

The divergent part of the boundary action (24) is given by

$$
\begin{aligned}
S_{b}= & \lim _{\rho \rightarrow 0} \int d^{d} x \frac{\rho^{1-\frac{d}{2}}}{l} \sqrt{-g_{(0)}}\left[\left(\frac{4 d^{3}+4 d^{2}}{l^{2}} \tilde{a}+\frac{4 d^{2}}{l^{2}} \tilde{b}+\frac{8 d}{l^{2}} \tilde{c}-\frac{2 d}{\tilde{\kappa}^{2}}+l \eta\right) \frac{1}{\rho}\right. \\
& +(-4 d \tilde{a}-2 \tilde{b}+\xi l) R_{(0)}+\left\{\frac{2 d^{3}-10 d^{2}+4 d}{l^{2}} \tilde{a}+\frac{2 d^{2}-8 d+4}{l^{2}} \tilde{b}+\frac{4 d-8}{l^{2}} \tilde{c}\right. \\
& \left.\left.+\frac{-d+2}{\tilde{\kappa}^{2}}+\frac{l}{2} \eta\right\} g_{(0)}^{i j} g_{(1) i j}+\mathcal{O}(\rho)\right] .
\end{aligned}
$$

Comparing (26) and (31), we find that the cancellation of the divergence requires

$$
\begin{aligned}
0 & =2 d \tilde{K} l^{2}+l \eta-2 K l^{2} \\
0 & =-4 d \tilde{a}-2 \tilde{b}+l \xi+\frac{l^{4} K}{d-2} \\
0 & =(2 d+2)(-4 d \tilde{a}-2 \tilde{b})-(-d+2) \tilde{K} l^{4}+\frac{l^{3} \eta}{2}+K l^{4} .
\end{aligned}
$$

Here

$$
\tilde{K} \equiv \frac{2 d^{2}+2 d}{l^{4}} \tilde{a}+\frac{2 d}{l^{4}} \tilde{b}+\frac{4}{l^{4}} \tilde{c}-\frac{1}{l^{2} \tilde{\kappa}^{2}} .
$$

Eq. 32) can be rewritten as follows:

$$
\tilde{K}=K-\frac{d+1}{l^{4}} A, \quad \eta=(2-2 d) K l-\frac{2 d(d+1)}{l^{3}} A, \quad \xi=-\frac{l^{3}}{d-2} K+\frac{A}{l} .
$$

Here

$$
A \equiv 4 d \tilde{a}+2 \tilde{b}
$$

The above equations seem not to be consistent with the result in [21], which requires $\tilde{K}=K$ even if $A \neq 0$. As the number of the independent parameters, $\tilde{a}, \tilde{b}, \tilde{c}, \tilde{\kappa}, \xi$ and $\eta$, is six but the number of the equations in (33) is three, there are three independent parameters. If we choose $\tilde{a}=\tilde{b}=\tilde{c}=0$, we find

$$
\frac{1}{\tilde{\kappa}^{2}}=-l^{2} K, \quad \eta=(2-2 d) K l, \quad \xi=-\frac{l^{3}}{d-2} K
$$


Especially in case of pure Einstein theory with cosmological term $(a=b=$ $c=0$ ), we find

$$
\frac{1}{\tilde{\kappa}^{2}}=\frac{1}{\kappa^{2}}, \quad \eta=\frac{(2 d-2)}{l \kappa^{2}}, \quad \xi=\frac{l}{(d-2) \kappa^{2}},
$$

This exactly corresponds to the counterterms in [13]. When we choose $\tilde{a}=$ $\tilde{b}=\tilde{c}=0$, the energy-momentum tensor defined by $S_{b}^{(2)}$ has the following form

$$
T_{i j} \equiv \frac{1}{\sqrt{-\tilde{g}}} \frac{\delta S_{b}^{(2)}}{\delta \hat{g}^{i j}}=\xi\left(\tilde{R}_{i j}-\frac{1}{2} \tilde{R} \hat{g}_{i j}\right)-\frac{\eta}{2} \hat{g}_{i j} .
$$

The expression in (38) coincides with the full energy-momentum tensor in [13] for the Einstein gravity.

Let us consider the holographic trace anomaly making the choice $\tilde{a}=\tilde{b}=$ $\tilde{c}=0$ in (36). First we consider $2 \mathrm{~d}$ case, where we should drop $\xi$ term since it is finite. In order to control the logarithmically divergent terms in the bulk action $S$, we choose $d-2=\epsilon<0$. Then the total action $S+S_{b}$ has the following form

$$
\begin{array}{r}
S+S_{b}=l^{3} K \int_{M_{d+1}} d^{d+1} x \frac{\rho^{1-\frac{\epsilon}{2}}}{2} \sqrt{-g_{(0)}}\left(-R_{(0)}-\frac{\epsilon}{l^{2}} g_{(0)}^{i j} g_{(1) i j}+\mathcal{O}(\rho)\right) \\
=l^{3} K \int_{M_{d}} d^{d} x\left[\frac{\rho_{0}^{1-\frac{\epsilon}{2}}}{\epsilon} \sqrt{-g_{(0)}}\left(-R_{(0)}-\frac{\epsilon}{l^{2}} g_{(0)}^{i j} g_{(1) i j}\right)+\mathcal{O}\left(\rho_{0}^{1-\frac{\epsilon}{2}}\right)\right]
\end{array}
$$

Here we replaced the integration over $M_{d+1}$ by

$$
\int_{M_{d+1}} d^{d+1} x \rightarrow \int_{M_{d}} d^{d} x \int_{\rho_{0}} d \rho
$$

with the finite (infrared) cutoff $\rho_{0}$, which is taken to be zero finally. We use the equation of the motion derived from (29) with respect to the variation over $g_{(1) i j}$

$$
\begin{aligned}
0 & =A R_{(0)}^{i j}+B g_{(0)}^{i j} R_{(0)}+2 C g_{(0)}^{i k} g_{(0)}^{j l} g_{(1) k l}+2 D g_{(0)}^{i j} g_{(0)}^{k l} g_{(1) k l} \\
A & \equiv l^{2} K+\frac{2 d-4}{l^{2}} b \\
B & \equiv-\frac{l^{2}}{2} K+\frac{4 d-4}{l^{2}} a+\frac{2}{l^{2}} b+\frac{4}{l^{2}} c
\end{aligned}
$$




$$
\begin{aligned}
C & \equiv \frac{3 d-10}{2} K+\frac{d^{2}-4 d+4}{l^{4}} b+\frac{4 d-8}{l^{4}} c \\
D & \equiv-\frac{3 d-8}{4} K+\frac{4 d^{2}-8 d+4}{l^{4}} a+\frac{3 d-4}{l^{4}} b+\frac{4}{l^{4}} c
\end{aligned}
$$

and solve the equation with respect to $g_{(1) i j}$. Just for $d=2$, we obtain

$$
g_{(1) i j}=-\frac{l^{2}}{4} R_{(0) i j}-\frac{l^{2}}{8} R_{(0)} g_{(0) i j}=-\frac{l^{2}}{4} R_{(0)} g_{(0) i j} .
$$

(Note that $R_{(0) i j}=\frac{1}{2} R_{(0)} g_{(0) i j}$ in 2 dimensions.) Therefore we obtain

$$
g_{(0)}^{i j} g_{(1) i j}=-\frac{l^{2}}{2}(1+\mathcal{O}(\epsilon))
$$

Since $\frac{\delta}{\delta g_{(0)}^{i j}}\left(\int_{M_{d}} d^{d} x \sqrt{-g_{(0)}} R_{(0)}\right)=\sqrt{-g_{(0)}}\left(R_{(0) i j}-\frac{1}{2} g_{(0) i j} R_{(0)}\right)$, of course, we obtain

$$
g_{(0)}^{i j} \frac{\delta}{\delta g_{(0)}^{i j}}\left(\int_{M_{d}} d^{d} x \sqrt{-g_{(0)}} R_{(0)}\right)=-\frac{\epsilon}{2} \sqrt{-g_{(0)}} R_{(0)} .
$$

Therefore only the leading term in (39) contributes to the trace anomaly and $g_{(0)}^{i j} g_{(1) i j}$ term, which gives the contribution of $\mathcal{O}(\epsilon)$, does not contribute. Then the trace anomaly for $d=2$ is given by

$$
T=\lim _{\epsilon \rightarrow 0-} \frac{2 \hat{g}_{(0)}^{i j}}{\sqrt{-\hat{g}_{(0)}}} \frac{\delta\left(S+S_{b}\right)}{\delta \hat{g}_{(0)}^{i j}}=l^{3} K R_{(0)},
$$

which is identical with the result found in [22] where another method was used. Note that there comes the contribution only from the bulk action. Although the equations of motion make the contribution vanish in the bulk but the additional contribution can appear from the surface due to the partial integration.

We now consider $d=4$ case. In this case we obtain,

$$
S+S_{b}=S_{\ln }+\text { finite terms . }
$$

As in $d=2$ case, we use dimensional regularization putting $d=4+\epsilon<0$. Eq. (41) can be solved with respect to $g_{(1) i j}$ just for $d=4+\epsilon$ as follows

$$
g_{(1) i j}=-\frac{l^{2}}{2} R_{(0) i j}+\frac{l^{2}}{24} R_{(0)} g_{(0) i j}+\mathcal{O}(\epsilon) .
$$


Substituting (47) into $S_{\ln }$ in (29) and using the regularization in (40), we obtain

$$
S_{l n}=\frac{1}{\epsilon} \int_{M_{d}} d^{d} x \sqrt{-g_{(0)}}\left\{\left(-\frac{l^{3}}{8 \kappa^{2}}+5 a l+b l\right)(G-F)+\frac{c l}{2}(G+F)+\mathcal{O}(\epsilon)\right\}
$$

Here we used the Gauss-Bonnet invariant $G$ and the square of the Weyl tensor $F$, which are given by

$$
\begin{aligned}
G & =R_{(0)}^{2}-4 R_{(0) i j} R_{(0)}^{i j}+R_{(0) i j k l} R_{(0)}^{i j k l} \\
F & =\frac{1}{3} R_{(0)}^{2}-2 R_{(0) i j} R_{(0)}^{i j}+R_{(0) i j k l} R_{(0)}^{i j k l} .
\end{aligned}
$$

In (48), $\mathcal{O}(\epsilon)$ term contains $g_{(0)}^{i j} g_{(2) i j}$ and other terms are a sum of the square of the curvatures $R_{(0)}^{2}, R_{(0) i j} R_{(0)}^{i j}$ and $R_{(0) i j k l} R_{(0)}^{i j k l}$. If we use the equation of motion, $g_{(0)}^{i j} g_{(2) i j}$ term can be given in terms of $g_{(0) i j}$. From the dimensional analysis, we can find that $g_{(0)}^{i j} g_{(2) i j}$ term is also given by a sum of the square of the curvatures and, if exists, the second order total derivatives of single curvature: $\square_{(0)} R_{(0)}$ or $D_{(0)}^{i} D_{(0)}^{j} R_{(0) i j}$. The total derivative terms can be dropped in the action due to the volume integration over $M_{d}$. Since

$$
\begin{aligned}
g_{(0)}^{i j} & \frac{\delta}{\delta g_{(0)}^{i j}}\left(\int_{M_{d}} d^{d} x \sqrt{-g_{(0)}} P_{(0)}\right) \\
& =-\frac{\epsilon}{2} \sqrt{-g_{(0)}} P_{(0)}+\left(\text { a sum of } \square_{(0)} R_{(0)} \text { and } D_{(0)}^{i} D_{(0)}^{j} R_{(0) i j}\right) \\
P_{(0)} & =R_{(0)}^{2}, R_{(0) i j} R_{(0)}^{i j}, R_{(0) i j k l} R_{(0)}^{i j k l},
\end{aligned}
$$

and the second order total derivatives of single curvature: $\square_{(0)} R_{(0)}$ and $D_{(0)}^{i} D_{(0)}^{j} R_{(0) i j}$, can be eliminated by a local counter terms if they appear in the trace anomaly, the $\mathcal{O}(\epsilon)$ terms in $(48)$ do not contribute to the anomaly. We obtain the following expression for the trace anomaly for $d=4$

$$
T=\left(-\frac{l^{3}}{8 \kappa^{2}}+5 a l+b l\right)(G-F)+\frac{c l}{2}(G+F) .
$$

The above result in (51) coincides with that in [22]. From here it is easy to get the holographic trace anomaly for Weyl gravity. Hence, we found the finite gravitational action (for asymptotically AdS spaces) in HD gravity in three 
and five dimensions by adding the local surface counterterm. This action correctly reproduces of holographic trace anomaly for dual (gauge) theory in $\mathrm{d} 2$ and $\mathrm{d} 4$. In principle, one can generalize all results for higher dimensions, say, d6, etc. With the growth of dimension, the technical problems become more and more complicated as the number of structures in boundary term is increasing.

\section{AdS black hole mass}

Now using the local surface counterterm prescription and the counterterm explicitly found in the previous section, we will find AdS black hole mass in HD gravity.

When the metric of the reference spacetime (e.g. AdS) has the following form

$$
d s^{2}=f(r) d r^{2}-N^{2}(r) d t^{2}+\sum_{i, j=1}^{d-1} \sigma_{i j} d x^{i} d x^{j},
$$

the mass $M$ of the black hole like object is given by

$$
M=\int d^{d-1} x \sqrt{\sigma} N \delta T_{t t}\left(u^{t}\right)^{2}
$$

Here $\delta T_{t t}$ is the difference of the $(t, t)$ component of the energy-momentum tensor in the spacetime with black hole like object from that in the reference spacetime and $u^{t}$ is the $t$ component of the unit time-like vector normal to the hypersurface given by $t=$ constant.

First we consider the case of $c=0$ in (1), where the Schwarzschild anti-de Sitter black hole is the exact solution:

$$
\begin{aligned}
d s_{\text {S-AdS }}^{2} & =-\mathrm{e}^{-2 \sigma} d t^{2}+\mathrm{e}^{2 \sigma} d r^{2}+\frac{r^{2}}{l^{2}} \sum_{i=1}^{d-1} \tilde{g}_{i j} d x^{i} d x^{j} \\
\mathrm{e}^{-2 \sigma} & =\frac{r^{2}}{l^{2}}\left(\frac{k r^{d-2}}{d-2}+\frac{\lambda^{2}}{d(d-1) r^{d}}-\mu\right) .
\end{aligned}
$$

Here $l$ is given by solving (28) after putting $c=0$ and $\tilde{g}_{i j}$ is the metric of $d-1$ dimensional Einstein manifold, where the Ricci tensor $\tilde{R}_{i j}$ given by $\tilde{g}_{i j}$ is proportional to $\tilde{g}_{i j}$ :

$$
\tilde{R}_{i j}=k \tilde{g}_{i j}
$$


We now consider the $d=4$ and $k=0$ case which appears in the throat limit of D3-brane. Then the $(t, t)$ component of the energy-momentum tensor in (38) has the following form:

$$
T_{t t}=\frac{\eta}{2} \hat{g}_{t t}=\frac{\eta}{2}\left(-\frac{r^{2}}{l^{2}}+\frac{r_{0}^{4}}{l^{2} r^{2}}\right) .
$$

Note that the Ricci tensor $\tilde{R}_{i j}$ and the scalar curvature $\tilde{R}$ in (38) vanish since $k=0$, which tells the boundary space is flat. Then we find

$$
\delta T_{t t}=T_{t t}-T_{t t}^{\mathrm{AdS}}=\frac{\eta r_{0}^{4}}{2 l^{2} r^{2}}, \quad T_{t t}^{\mathrm{AdS}}=-\frac{\eta r^{2}}{2 l^{2}} .
$$

For the reference AdS metric, we find

$$
\sqrt{\sigma} N=\frac{r^{2}}{l^{2}}
$$

Then substituting (57) and (58) into (53), the mass $M$ is

$$
M=\frac{\eta r_{0}^{4}}{2 l^{4}} \int d^{3} x
$$

which agrees with the result obtained by using the standard prescription in [23]. Especially, in the Einstein gravity case $a=b(=c)=0$, it reproduces the standard prescription result in [24, 25]:

$$
M=\frac{3 r_{0}^{4}}{l^{5} \kappa^{2}} \int d^{3} x
$$

We now consider the Weyl gravity (5). In this model, AdS is the exact solution and from (28) we find its radius is identical with that of the Einstein gravity:

$$
l^{2}=-\frac{12}{\Lambda \kappa^{2}} .
$$

The Schwarzschild-AdS (S-AdS) solution is not, however, the exact solution of the system. Assuming $\hat{c}$ is small, we can solve S-AdS type solution perturbatively :

$$
\begin{aligned}
\mathrm{e}^{-2 \sigma} & =\frac{l^{2}}{r^{2}}\left(1-\frac{r_{0}^{4}}{r^{4}}+2 \epsilon \frac{r_{0}^{8}}{r^{8}}\right) \\
\epsilon & \equiv \frac{\hat{c} \kappa^{2}}{l^{2}} .
\end{aligned}
$$


Here we make the coordinate choice as in (54) :

$$
d s^{2}=-\mathrm{e}^{-2 \sigma} d t^{2}+\mathrm{e}^{2 \sigma} d r^{2}+\frac{r^{2}}{l^{2}} \sum_{i=1}^{d-1} \eta_{i j} d x^{i} d x^{j}
$$

As the difference of the asymptotic behavior from that of the S-AdS solution in the Einstein gravity appears only in the next-to-next order in $\frac{1}{r^{4}}$, we can evaluate the mass $M$ in the way similar to $c=0$ case in (59)

$$
M=\frac{\eta r_{0}^{4}}{2 l^{4}} \int d^{3} x=\frac{3 r_{0}^{4}}{l^{5} \kappa^{2}} \int d^{3} x
$$

In (64), the $\epsilon(\hat{c})$ correction does not appear and the result does not seem to agree with the standard prescription result in [23], where the mass (energy) is given by

$$
M=\frac{3(\pi T) l^{3}}{\kappa^{2}}(1+18 \epsilon) \int d^{3} x .
$$

Here the temperature $T$ is given by

$$
\pi T=\frac{r_{0}}{l^{2}}\left(1-\frac{5}{2} \epsilon\right)
$$

Therefore we obtain

$$
M=\frac{3 r_{0}^{4}}{l^{5} \kappa^{2}}(1+8 \epsilon) \int d^{3} x
$$

In (67), there appear $\epsilon$ corrections in the next-to-leading order, which conflict with (65). This expresses the ambiguity in $R^{2}$ gravity in the choice of regularizations (standard prescription or local counterterm prescription) to make the action to be finite. In fact, we have chosen $\tilde{a}=\tilde{b}=\tilde{c}=0$ in Eq. (36) in order to fix the ambiguities in Eq.(34). For general case when $\tilde{a}, \tilde{b}$ and $\tilde{c}$ do not vanish, $\eta$ in (34) is shifted by $A$ in (35). Then we obtain, instead of (64),

$$
M=\frac{\eta r_{0}^{4}}{2 l^{4}} \int d^{3} x=\frac{3 r_{0}^{4}}{l^{5} \kappa^{2}}\left(1-\frac{20 \kappa^{2}}{3 l^{2}} A\right) \int d^{3} x .
$$

Therefore the expression in (67) can be reproduced if we choose

$$
-\frac{20 \kappa^{2}}{3 l^{2}} A=8 \epsilon
$$


or using the expression for $\epsilon$ in (62)

$$
A=-\frac{6}{5} \hat{c}
$$

Then for general case with $c \neq 0$, it would be natural to choose

$$
A=-\frac{6}{5} c
$$

that is, in terms of $\eta$ and $\xi$ in (34) for $d=4$,

$$
\eta=-6 l\left(\frac{40 a}{l^{4}}+\frac{8}{l^{4}}-\frac{4 c}{l^{4}}-\frac{1}{l^{2} \kappa^{2}}\right), \quad \xi=-\frac{l^{3}}{2}\left(\frac{40 a}{l^{4}}+\frac{8}{l^{4}}+\frac{8 c}{5 l^{4}}-\frac{1}{l^{2} \kappa^{2}}\right) .
$$

Even if we choose $A$ as in (71), of course, the previous results when $c=0$ do not change.

As a check, we consider $a=b=0$ but $c \neq 0$ case. By the calculation similar to the previous case of the square of the Weyl tensor, we obtain

$$
M=\frac{\eta r_{0}^{4}}{2 l^{4}} \int d^{3} x=\frac{3 r_{0}^{4}}{l^{4}}\left(1+\frac{4 \kappa^{2} c}{l^{2}}\right) \int d^{3} x .
$$

Note that from (72), $\eta$ is now given by

$$
\eta=-\frac{6}{l \kappa^{2}}\left(1+\frac{4 c \kappa^{2}}{l^{2}}\right) .
$$

The result in (73) agrees with the result obtained by using standard prescription in [23]. In [23], the corresponding eq. is given by

$$
\begin{aligned}
& M=E=\frac{3 V_{3}(\pi T)^{4}}{\kappa^{2}}\left(1+12 c \kappa^{2}\right) \\
& \frac{1}{\kappa^{2}}=\frac{N^{2}}{4 \pi^{2}}, \quad c \kappa^{2}=\frac{1}{16}, \quad V_{3}=\int d^{3} x .
\end{aligned}
$$

Here $l$ is chosen to be unity: $l=1$. Since the temperature $T$ is given by

$$
\pi T=r_{0}\left(1-2 c \kappa^{2}\right)
$$

we find

$$
M=\frac{3 V_{3} r_{0}^{4}}{\kappa^{2}}\left(1+4 c \kappa^{2}\right)
$$


which is identical with (73) if we put $l=1$.

Hence, we showed how for general HD gravity by fixing of unspecified parameters of local surface counterterm to make the complete correspondence between standard prescription and local surface counterterm prescription (at least, in asymptotically AdS spaces). One can now repeat the similar calculation for another asymptotically AdS spaces, like Kerr-AdS one, etc.

\section{Discussion}

In summary, in this paper we constructed the local surface counterterm for HD gravity and for Weyl (stringy) gravity. Its coefficients are defined for general three- and five-dimensional HD gravity. Adding the local surface counterterm to bulk action of HD gravity makes the complete gravitational action to be finite (at least for asymptotically AdS spaces). The gravitational energy-momentum tensor is getting also to be well-defined. As a by-product we obtain the holographic conformal anomaly corresponding to $\mathrm{d} 2$ and $\mathrm{d} 4$ dual boundary quantum field theory (via AdS/CFT correspondence). This result coincides with the previous calculation made by different method. The calculation of AdS black hole mass for HD gravity is made, using local surface counterterm prescription. The corresponding mass (energy) for AdS space under consideration coincides with the standard prescription result.

Hence, our results indicate to universality of local surface counterterm prescription developed originally for Einstein gravity. It is very interesting to note that there are immediate directions on extension of our results. First of all, one could consider higher dimensional versions (say seven-dimensional gravity) and to construct the corresponding local surface counterterm and finite gravitational action in this case. This may be useful in construction (via AdS/CFT correspondence) of dual six-dimensional quantum field theory (or, at least in consideration of next-to-leading corrections in such theory). Second, we limited our discussion to asymptotically AdS spaces. Clearly, one can generalize the local surface counterterm in HD gravity in the way indicated in ref. [13] for Einstein gravity in order to make the gravitational action to be finite in asymptotically flat (or other non-compact) spaces. Third, having the correct boundary term in HD gravity one may reconsider the circle of problems related with the role of higher derivative gravitational terms to early Universe (for example, minisuperspace model). This will be considered 
elsewhere.

Acknowledgments. The work by SDO has been partially supported by RFBR project N99-02-16617.

\section{References}

[1] O. Aharony, S.S. Gubser, J. Maldacena, H. Ooguri and Y. Oz, hepth/9905111.

[2] V. Balasubramanian and P. Kraus, hep-th/9902121.

[3] G.W. Gibbons and S.W. Hawking, Phys.Rev. D15 (1977) 2752.

[4] J.D. Brown and J.W. York, Phys.Rev. D47 (1993) 1407.

[5] L.F. Abbott and S. Deser, Nucl.Phys. B195 (1982) 76.

[6] A. Ashtekar and A. Magnon, Class.Quant.Grav. 1 (1984) L39.

[7] M. Henneaux and C. Teitelboim, Comm.Math.Phys. 98 (1985) 391.

[8] J.D. Brown, J. Creighton and R. Mann, Phys.Rev. D50 (1994) 6394.

[9] G.T. Horowitz and S.W. Hawking, Class.Quant.Grav. 13 (1996) 1487.

[10] G.T. Horowitz and R.C. Myers, Phys.Rev. D59 (1999) 026005.

[11] R. Emparan, C.V. Johnson and R.C. Myers, hep-th/9903238.

[12] A. De Benedictis and K.S.Wiswanathan, hep-th/9911060.

[13] P. Kraus, F. Larsen and R. Siebelink, hep-th/9906127.

[14] J. Ho, hep-th/9910124.

[15] I.L. Buchbinder, S.D. Odintsov and I.L. Shapiro, Effective Action in Quantum Gravity, IOP Publishing, Bristol and Philadelphia 1992.

[16] A. Fayyazuddin and M. Spalinski, Nucl.Phys. B535 (1998) 219, hepth/9805096; O. Aharony, A. Fayyazuddin and J.M. Maldacena, JHEP 9807 (1998) 013, hep-th/9806159. 
[17] A.A. Tseytlin, Nucl.Phys. B467 (1996) 383.

[18] D.J. Gross and E. Witten, Nucl.Phys. B277 (1986) 1; A.A. Tseytlin, Phys.Lett. B176 (1986) 92.

[19] M.T. Grisaru and D. Zanon, Phys.Lett. B177 (1986) 347; M.D. Freeman, C.N. Pope, M.F. Sohnius and K.S. Stelle, Phys.Lett. B178 (1986) 199.

[20] S. Nojiri and S.D. Odintsov, hep-th/9910113.

[21] S.W. Hawking and J.C. Luttrell, Nucl.Phys. B247 (1984) 250.

[22] S. Nojiri and S.D. Odintsov, hep-th/9903033, to appear in Int.J.Mod.Phys. A.

[23] S. Nojiri and S.D. Odintsov, hep-th/9908065, to appear in Phys.Lett. B.

[24] S. Gubser, I. Klebanov and A. Tseytlin, Nucl.Phys. B534 (1998) 202, hep-th/9805156.

[25] S. Gubser, I. Klebanov and A. Peet, Phys.Rev. D54 (1996) 3915. 